\title{
ARMAZENAMENTO DE PITANGA SOB ATMOSFERA MODIFICADA E REFRIGERAÇÃO: I-TRANSFORMAÇÕES QUÍMICAS EM PÓS-COLHEITA ${ }^{1}$
}

\author{
ADRIANA FERREIRA DOS SANTOS², SILVANDA DE MELO SILVA³, RICARDO ELESBÃO ALVES ${ }^{4}$
}

\begin{abstract}
RESUMO - Este experimento teve como objetivo avaliar as transformações pós-colheita em pitangas colhidas nos estádios de maturação vermelhoalaranjado (VA) e vermelho predominante (VP) e mantidas sob atmosfera modificada (AM) por filme de cloreto de polivinila (PVC), a $10 \pm 0,5^{\circ} \mathrm{C}, 14$ $\pm 0,5^{\circ} \mathrm{C}$ e $(90 \pm 1 \% \mathrm{UR})$ e a temperatura ambiente $\left(23 \pm 2^{\circ} \mathrm{C}\right.$ e $\left.85 \pm 2 \% \mathrm{UR}\right)$. O uso de $\mathrm{AM}$ associada à refrigeração permitiu manutenção dos sólidos solúveis, acidez titulável, dos açúcares solúveis totais e vitamina $\mathrm{C}$, e também resultou em menor taxa de aumento nos carotenóides totais para frutos do estádio de maturação VA mantido a 10 e $14^{\circ} \mathrm{C}$. Em conjunto, pitangas colhidas no estádio vermelho-alaranjado foram as que apresentaram melhor manutenção das características intrínsecas durante oito dias de armazenamento sob atmosfera modificada a $10^{\circ} \mathrm{C}$.
\end{abstract}

Termos para Indexação: Eugenia uniflora, vitamina C, clorofila e carotenóides totais.

\section{STORAGE OF SURINAME CHERRY UNDER MODIFIED ATMOSPHERE AND REFRIGERATION: I - POSTHARVEST CHEMICAL CHANGES}

\begin{abstract}
The aim of this experiment was to evaluate postharvest changes in Suriname Cherry harvested in the maturity stages red-orangish (RO) and predominant red (PR), kept under modified atmosphere (MA) by polyvinyl chloride film (PVC), at $10 \pm 0.5^{\circ} \mathrm{C}, 14 \pm 0.5^{\circ} \mathrm{C}, 90 \pm 1 \% \mathrm{RH}$ and room temperature $\left(23 \pm 2^{\circ} \mathrm{C}\right)$. The use of MA associated with refrigeration allowed the maintenance of total soluble solids, titratable acidity, soluble sugars, and vitamin $\mathrm{C}$, and also resulted in lower increase rate in total carotenoids for fruits in the RO maturity stages kept at 10 e $14^{\circ} \mathrm{C}$. All together, Suriname Cherries harvested in red-orangish skin color were those that presented better maintenance of the intrinsic characteristics during 8 days storage under modified atmosphere at $10^{\circ} \mathrm{C}$.
\end{abstract}

Index terms: Eugenia uniflora, vitamin C, total chlorophyll and carotenoids.

\section{INTRODUÇÃO}

A pitangueira (Eugenia uniflora L.) é uma mirtácea tipicamente brasileira, cujo fruto vem crescendo em importância econômica no Nordeste. O consumo de pitanga vem aumentando no Nordeste e no Brasil (Kavati, 1996), estabelecendo-se o interesse para exportação desse fruto (Santos et al., 2003).

O armazenamento visa, sobretudo, minimizar a intensidade dos processos vitais dos frutos, através da utilização de condições adequadas que permitam redução da taxa do metabolismo normal, sem alterações na fisiologia dos frutos (Kader, 1986), principalmente daqueles frutos altamente perecíveis, como a pitanga (Santos et al., 2002). A redução da temperatura de armazenamento é o meio mais eficiente em prolongar a vida útil de produtos colhidos (Kays, 1997); no entanto, a sensibilidade a baixas temperaturas pode resultar em danos pelo frio, restringindo o tempo de armazenamento e a vida útil pós-colheita de frutos (Morris, 1982). A modificação da atmosfera tem sido bastante utilizada com o objetivo de minimizar as perdas e prolongar a vida útil pós-colheita de frutos e hortaliças (Jaime et al., 2001). O estabelecimento de uma composição gasosa diferente da do ar, pela redução do nível de $\mathrm{O}_{2}$ e elevação de $\mathrm{CO}_{2}$ no interior da embalagem, pode resultar na redução da atividade metabólica do produto (Beaudry, 2004), sendo mais evidenciada quando associada à refrigeração (Kays, 1997). Na maioria dos vegetais, o uso apropriado de atmosfera modificada (AM) reduz a taxa respiratória e a produção de etileno, retarda o amaciamento da polpa e as mudanças fisiológicas (Kader, 1986). A atmosfera modificada requer baixo custo operacional, entretanto o seu emprego exige conhecimento da fisiologia do fruto (estádio de maturação, dependência a $\mathrm{CO}_{2}$ e $\mathrm{O}_{2}$ ), das características do filme flexível e fatores ambientais de armazenamento, tais como temperatura e umidade relativa (Beaudry, 2004).

O objetivo deste trabalho foi avaliar a influência do estádio de maturação e do emprego de atmosfera modificada sobre as transformações químicas pós-colheita de pitanga armazenada a 10 e a $14^{\circ} \mathrm{C}$ e sob condições ambientes $\left(23 \pm 2^{\circ} \mathrm{C}\right.$ e $\left.85 \pm 2 \% \mathrm{UR}\right)$.

\section{MATERIALE MÉTODOS}

Os frutos utilizados foram provenientes de um grupo de plantas localizadas no pomar da Embrapa Algodão-CNPA(Centro Nacional de Pesquisa e Algodão), município de Campina Grande-PB. Após a colheita manual, os frutos foram acondicionados em caixas isotérmicas e transportados para o laboratório, onde foram selecionados quanto ao tamanho, peso, estádio de maturação e ausência de desordens fisiológicas ou doenças. Como tratamento antifúngico, os frutos foram imersos, por 10 minutos, em uma solução de hipoclorito de sódio comercial a $0,5 \%$ e, em seguida, enxaguados com água destilada e secos ao ar. Os frutos, selecionados em dois estádios de maturação (vermelhoalaranjado (VA) e predominantemente vermelho (VP)), foram armazenados sob AM após, aproximadamente, $12 \mathrm{~h}$ da colheita.

$\mathrm{Na}$ instalação do experimento, 24 frutos (aproximadamente $300 \mathrm{~g}$ ) foram acondicionados em bandejas de poliestireno expandido $(250 \times 150 \times 25 \mathrm{~mm})$. Em seguida, metade do total das bandejas foi envolvida com filme de cloreto de polivinila (PVC) flexível de $13 \mu \mathrm{m}$ de espessura, para a modificação da atmosfera. As condições de armazenamento utilizadas foram câmara fria a $10 \pm 0,5^{\circ} \mathrm{C}$ e $90 \pm 1 \% \mathrm{UR}$, câmara incubadora BOD, a $14 \pm 0,5^{\circ} \mathrm{C}$ e $90 \pm 1 \%$ UR. Paralelamente, foi instalado um experimento similar sob condições ambientes $\left(23 \pm 2^{\circ} \mathrm{C}\right.$ e $85 \pm 2 \%$ UR). Para a temperatura ambiente, foram feitas avaliações diárias até o $5^{\circ}$ dia e, para frutos mantidos a 10 e $14^{\circ} \mathrm{C}$, as avaliações foram realizadas a cada 2 dias, durante 10 dias. As avaliações realizadas foram: sólidos solúveis (\%), $\mathrm{pH}$, acidez titulável (\% de ácido cítrico), açúcares solúveis totais $(\mathrm{g} / 100 \mathrm{~g})$, vitamina $\mathrm{C}$ total $(\mathrm{mg} / 100 \mathrm{~g})$, clorofila total $(\mathrm{mg} /$ $100 \mathrm{~g}$ ) e carotenóides totais (mg/100g) (Association, 1989).

$\mathrm{O}$ experimento foi instalado em delineamento inteiramente casualizado, em esquema fatorial ( 2 × 2 x 5), com três repetições. Cada temperatura de armazenamento foi avaliada individualmente. Os fatores estudados foram dois estádios de maturação (VA e VP), modificação da atmosfera em dois níveis (AA (atmosfera ambiente) e AM (atmosfera modificada), e cinco períodos de avaliação. A partir dos resultados das análises de variância preliminares, e verificando-se a interação entre os

\footnotetext{
${ }^{1}$ (Trabalho 174/2004). Recebido: 08/11/2004. Aceito para publicação: 19/01/2006. Parte do trabalho de Dissertação do Mestrado em Agronomia do primeiro autor. ${ }^{2}$ Eng. Agr. M.Sc em Agronomia, Aluno de Doutorado do Programa de Pós-Graduação em Agronomia, CCA/UFPB, Caixa Postal 04, adre.santos@bol.com.br. ${ }^{3}$ Prof. Ph.D., DCFS/CCA/UFPB, Caixa Postal 04, CEP.: 58397-970, Areia, PB, silvasil@cca.ufpb.br.

${ }^{4}$ Pesq. Dr., Embrapa-CNPAT, Caixa Postal 3711, Fortaleza-CE, elesbao@cnpat.embrapa.br.
} 
fatores, o tempo foi desdobrado dentro de cada tratamento, e os resultados submetidos à análise de regressão polinomial. Foram consideradas equações de até $2^{\circ}$ grau. O coeficiente de determinação mínimo para utilização das curvas foi de 0,60 . Para os casos em que a interação entre os fatores estudados não foi significativa, os valores foram representados pelas médias dos tratamentos sem o ajuste de curvas.

\section{RESULTADOS E DISCUSSÃO}

O conteúdo de sólidos solúveis (SS) foi mais baixo para frutos do estádio $\mathrm{AV}$ mantidos a 10 e $14^{\circ} \mathrm{C}$ e para os do estádio VP a $10^{\circ} \mathrm{C}$, sob AM, quando comparado aos mantidos em AA (Fig. 1). Estes resultados devem-se, provavelmente, ao fato de que, sob condições de AM, os processos de amadurecimento e metabolização dos açúcares ocorrem mais lentamente (Wills et al., 1989). Em geral, o aumento do conteúdo dos SS está acompanhado do declínio do conteúdo de ácidos e da conversão dos mesmos para açúcares na via glicolítica (Kays, 1997). Valores elevados de SS observados nos frutos sob AA podem ser atribuídos ao avanço dos processos de amadurecimento (Santos et al., 2003), bem como à perda completa da turgescência, com conseqüente concentração da polpa. Pitangas no estádio de maturação VA, quando mantidas sob AM, apresentaram menor variação no conteúdo de SS ao final do período de armazenamento, sobretudo a $10^{\circ} \mathrm{C}$.

Com relação aos valores de $\mathrm{pH}$, houve diferença significativa $(\mathrm{P} \leq 0,01)$ para as interações estádio $\mathrm{x}$ atmosfera $\mathrm{x}$ períodos de armazenamento, apenas para as pitangas mantidas sob refrigeração. Observou-se que os valores de pH (Fig. 2A), durante o período de armazenamento, não apresentaram diferença entre os tratamentos avaliados, independentemente da temperatura. A pequena variação observada para $\mathrm{pH}$, independentemente dos estádios de maturação, não correspondeu aos resultados observados para AT, indicando a ocorrência de um sistema tampão eficiente em manter o $\mathrm{pH}$, mesmo com alteração na acidez.
Verificou-se que os frutos mantidos sob atmosfera modificada apresentaram menor acidez titulável (AT), exceto para frutos do estádio VP mantidos sob AM a $23^{\circ} \mathrm{C}$, do $2^{\circ}$ ao $4^{\circ}$ dia de armazenamento. AAT de pitangas mantidas sob AA, em geral, tendeu a aumentar ao final do armazenamento, como conseqüência do avanço da maturação dos frutos. Pitangas no estádio de maturação VA, mantidas sob $\mathrm{AM}$ a $10^{\circ} \mathrm{C}$, mantiveram o teor de AT constante (Fig. 2B). Os valores mais baixos de AT observados nos frutos mantidos sob AM, durante o armazenamento, podem ser explicados pela diminuição nos níveis de $\mathrm{O}_{2}$ e respectivo aumento nos de $\mathrm{CO}_{2}$, provocados pela AM, o que pode ter resultado na diminuição da taxa de atividade de enzimas relacionadas ao metabolismo respiratório (Zagory e Kader, 1988). Com a evolução da concentração de $\mathrm{CO}_{2}$ e da redução de $\mathrm{O}_{2}$, no armazenamento sob AM, os sistemas enzimáticos de degradação da glicose no processo respiratório podem ser afetados, provocando alterações no ciclo de Krebs. Dessa forma, devido à redução da taxa respiratória, em decorrência da AM, pode ter ocorrido uma redução da atividade da succínico desidrogenase, enzima diretamente afetada pela redução do nível de $\mathrm{O}_{2}$ (Salunkhe e Wu, 1974), resultando em menores níveis de AT, como observado por Wallace (1966) em limões.

Observaram-se teores mais elevados de açúcares solúveis totais (AST) em pitangas do estádio PV (Fig. 3A). Verificou-se também uma tendência de pequeno aumento nos conteúdos de AST nos frutos sob AA mantidos a $10^{\circ} \mathrm{C}$, o que pode estar relacionado com a maior perda de água sofrida por esses frutos. Pitangas no estádio VA, mantidas sob $\mathrm{AM}$ a $10^{\circ} \mathrm{C}$ e do estádio $\mathrm{VP}$ a $14^{\circ} \mathrm{C}$ apresentaram pouca variação no teor de AST durante o armazenamento, indicando que esses estádios de maturação podem ter se ajustado satisfatoriamente à temperatura de armazenamento e à modificação da atmosfera. O efeito positivo da $\mathrm{AM}$, nos estádios de maturação e nas temperaturas avaliadas, deve-se provavelmente ao aumento da concentração de $\mathrm{CO}_{2}$ e redução da concentração de $\mathrm{O}_{2}$ no interior da embalagem como um fator que possivelmente altera o fluxo de carbono na glicólise (Kader, 1986), reduzindo e modificando o metabolismo dos açúcares (Hansen e

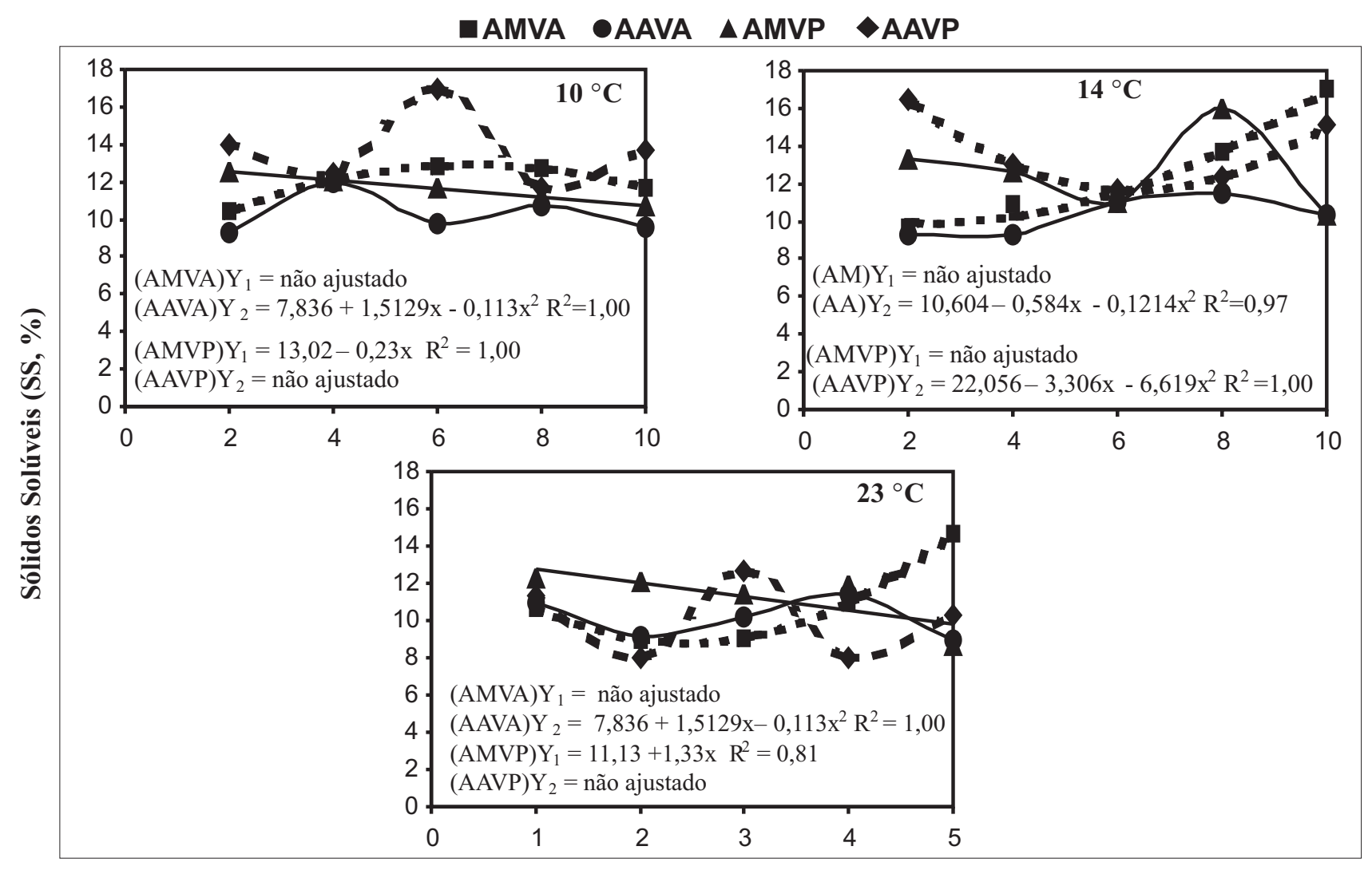

Período pós-colheita (dias)

FIGURA 1 - Conteúdo de Sólidos Solúveis (SS) em pitanga, nos estádios de maturação vermelho-alaranjado (AV) e vermelho predominante (PV), durante o armazenamento sob atmosfera modificada $\left(\mathrm{AMY}_{1} \longrightarrow\right.$ ) e ambiente $\left(\mathrm{AAY}_{2} \longrightarrow\right)$ a 10 e a $14^{\circ} \mathrm{C}$ e sob condições ambientes $\left(23 \pm 2^{\circ} \mathrm{C}\right)$. 


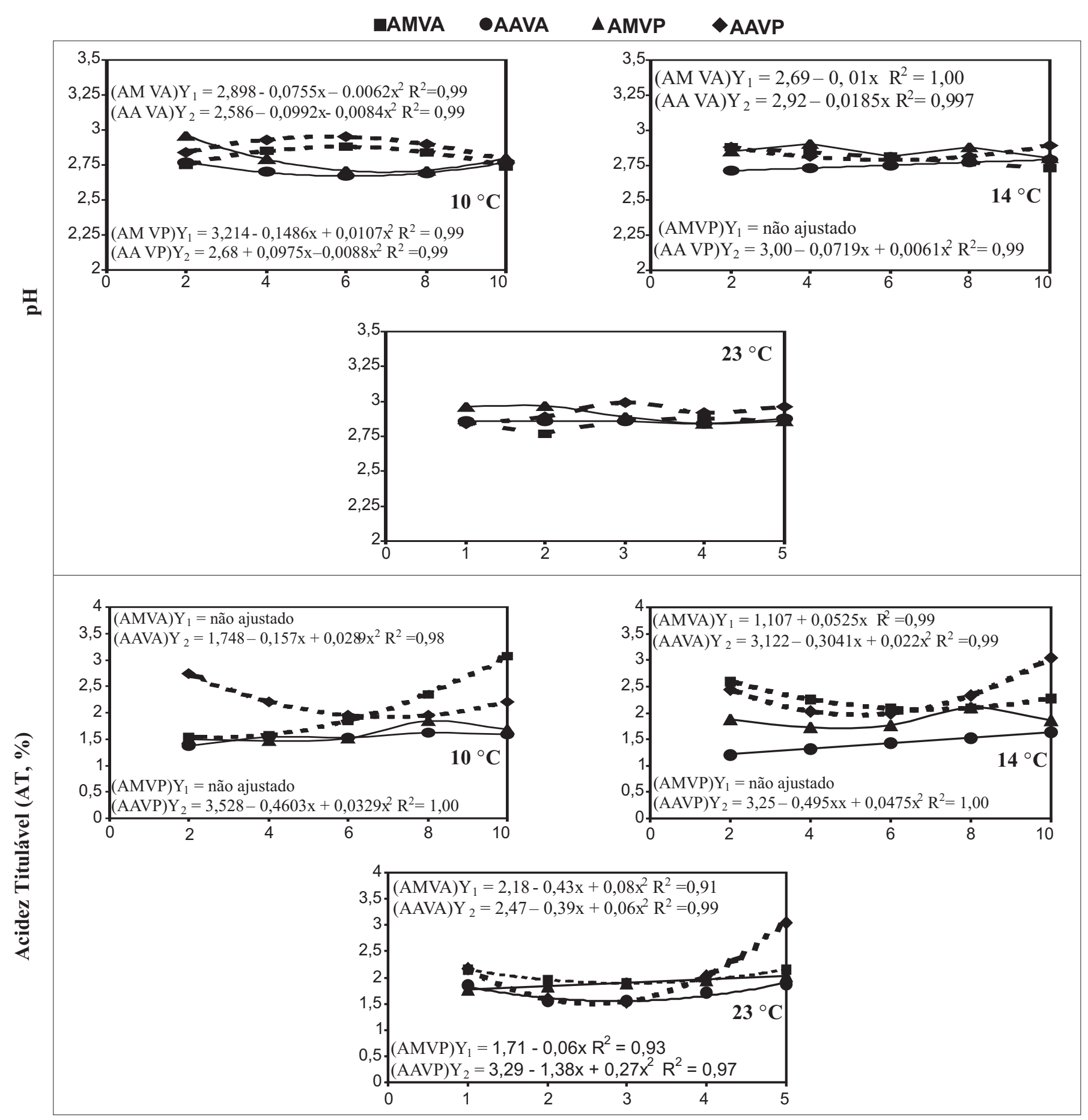

Período pós-colheita (dias)

FIGURA 2 - pH(A) e AT(B) em pitanga, nos estádios de maturação vermelho-alaranjado(AV) e vermelho predominante(PV), durante o armazenamento sob atmosfera modificada $\left(\mathrm{AMY} \longrightarrow\right.$ ) e ambiente $\left(\mathrm{AAY}_{2} \rightarrow\right.$ ) a 10 e a $14^{\circ} \mathrm{C}$ e sob condições ambientes $\left(23 \pm 2^{\circ} \mathrm{C}\right)$.

Weichmann, 1987; Saez et al., 1999).

O uso de AM notadamente manteve os conteúdos de vitamina C mais elevados quando comparados a AA (Fig 3B). Pitangas armazenadas sob condições ambientes apresentaram maiores teores de vitamina $\mathrm{C}$ do que as mantidas sob refrigeração. Isso ocorreu, provavelmente, em decorrência da concentração do suco celular resultante da maior perda de água nessa temperatura (Kays, 1997). Os conteúdos de vitamina $\mathrm{C}$, independentemente da temperatura de armazenamento e do estádio de maturação, diminuíram durante o armazenamento, sobretudo sob AA. Para os frutos sob refrigeração, as reduções mais acentuadas ocorreram a $14^{\circ} \mathrm{C}$, principalmente no estádio VA.

$\mathrm{O}$ uso de $\mathrm{AM}$ proporcionou maior retenção de clorofila $(\mathrm{P} \leq$ 0,05 ) quando comparado a AA(Fig.4A), independentemente dos estádios de maturação. O efeito da AM, na maior retenção da clorofila, pode ser resultante da mais elevada concentração de $\mathrm{CO}_{2}$ e mais baixa de $\mathrm{O}_{2}$ em reduzir as taxas metabólicas (Kays, 1997) e, conseqüentemente, reduzir a atividade das enzimas clorofilase, peroxidase e polifenoloxidase (Kader, 1986).

O desenvolvimento dos carotenóides totais (Fig. 4B) durante o armazenamento de pitangas mantidas sob AM foi inferior ao dos frutos sob AA, independentemente dos estádios de maturação. Esse comportamento, provavelmente, deve-se ao efeito da alta concentração de $\mathrm{CO}_{2}$ e redução de $\mathrm{O}_{2}$ proporcionada pela $\mathrm{AM}$, na redução da biossíntese dos carotenóides (Kader, 1986). Conteúdos mais elevados de carotenóides totais foram observados para frutos predominantemente vermelhos, independentemente da atmosfera e temperatura de armazenamento. 


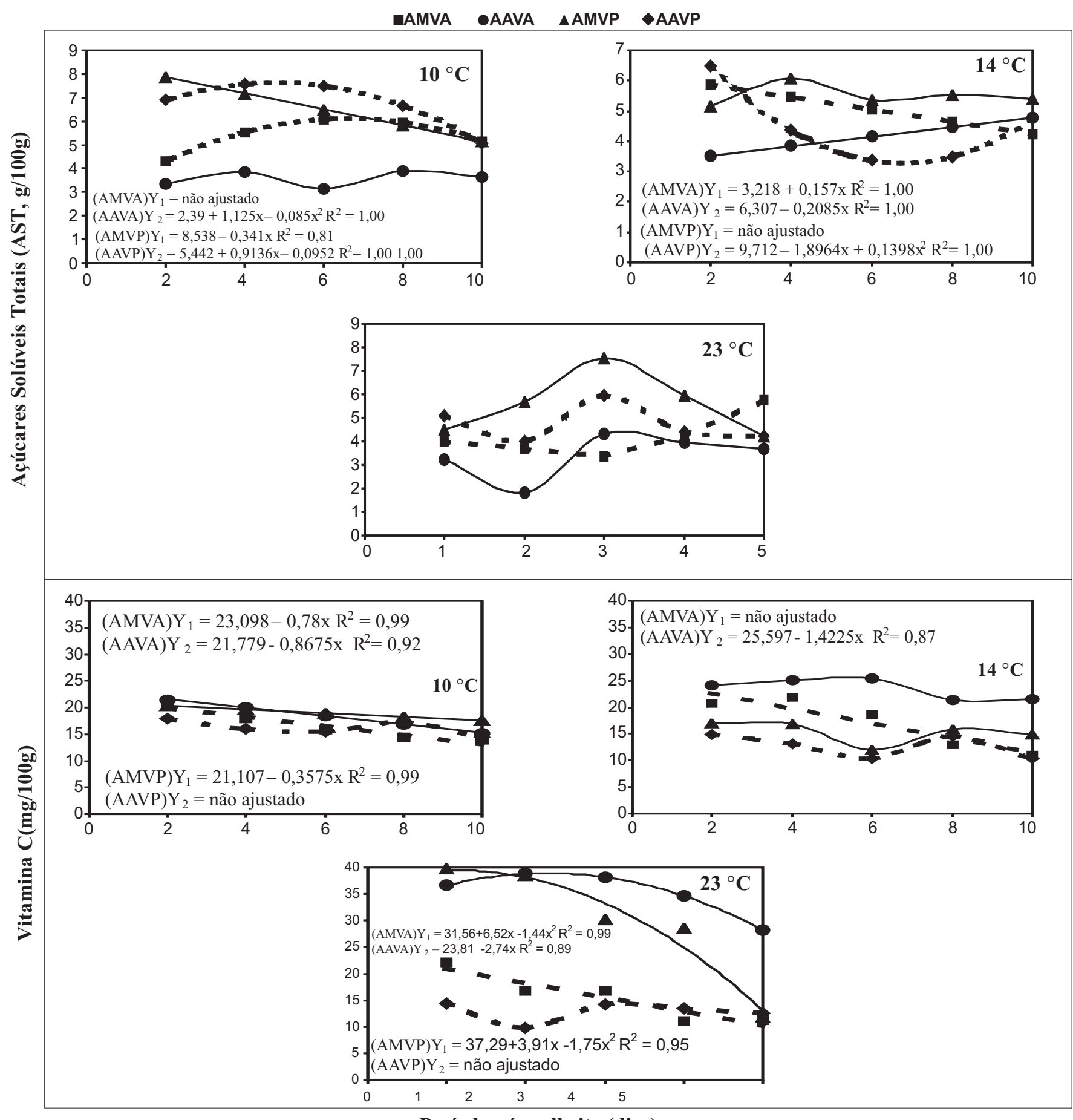

Período pós-colheita (dias)

FIGURA 3 - AST (A) e Vitamina C (B) em pitanga, nos estádios de maturação vermelho-alaranjado (AV) e vermelho predominante (PV), durante o armazenamento sob atmosfera modificada $\left(\mathrm{AMY}_{1} \longrightarrow\right)$ e ambiente $\left(\mathrm{AAY}_{2} \longrightarrow\right)$ ) a 10 e a $14^{\circ} \mathrm{C}$ e sob condições ambientes $\left(23 \pm 2^{\circ} \mathrm{C}\right)$. 


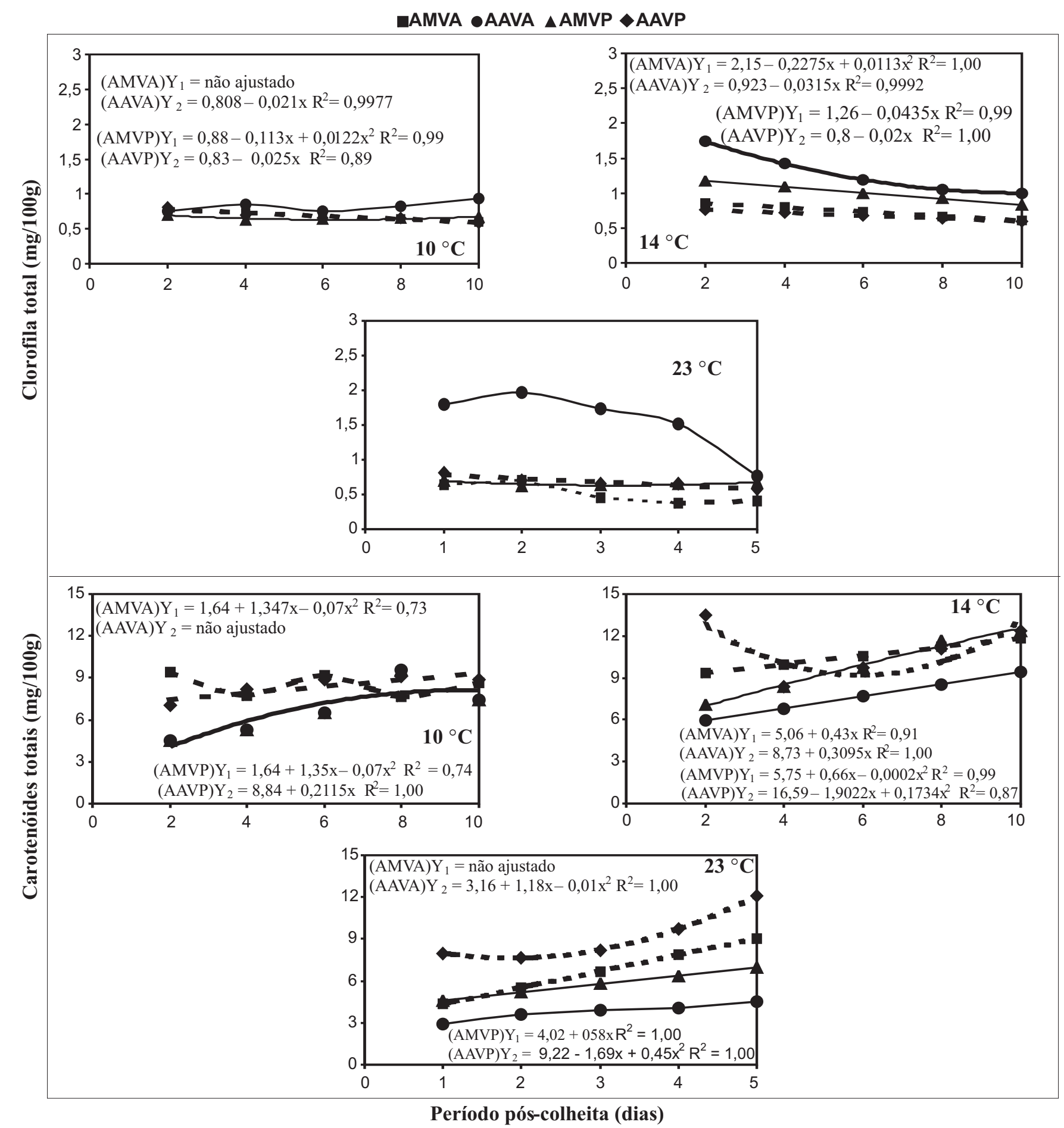

FIGURA 4 - Clorofila total (A) e Carotenóides totais (B) em pitanga, nos estádios de maturação vermelho-alaranjado (AV) e vermelho predominante $(\mathrm{PV})$, durante o armazenamento sob atmosfera modificada $\left(\mathrm{AMY} \longrightarrow\right.$ ) e ambiente $\left(\mathrm{AAY}_{2} \longrightarrow\right.$ a 10 e a $14^{\circ} \mathrm{C}$ e sob condições ambientes $\left(23 \pm 2^{\circ} \mathrm{C}\right)$.

\section{CONCLUSÕES}

1. A temperatura de $10^{\circ} \mathrm{C}$ mostrou-se a mais eficiente para a manutenção das características químicas de pitangas, e o estádio de maturação vermelho-alaranjado (VA) o mais adequado ao armazenamento, mantendo as características avaliadas durante oito dias.

2. O uso de atmosfera modificada resultou na manutenção, durante um período mais prolongado, da acidez titulável, vitamina $\mathrm{C}$, clorofila e carotenóides totais, para frutos mantidos a $10^{\circ} \mathrm{C}$ e a $14^{\circ} \mathrm{C}$, independentemente do estádio de maturação.

\section{AGRADECIMENTOS}

Os autores agradecem ao CNPq pelo suporte financeiro deste experimento.

\section{REFERÊNCIAS}

ASSOCIATION OF OFFICIAL ANALYTICAL CHEMISTS. Official methods of analysis. Washington, DC., 1984. 1989p.

BEAUDRY, R. M. Modified atmosphere packaging. In: HARDENBURG, R. E; WATADA, A. E.; WANG, C. Y.; GROSS, K. The Commercial storage of fruits, vegetables, and florists, and nursery stocks. Washington, D.C., 2004, p. 456-567.

HANSEN, H.; WEICHMAN, J. Carbohydrates. In: WEICHMANN, J. Postharvest physiology of vegetables. New York: Marcel Dekker, 1987. cap.23, p.113-70.

KADER, A. A. Biochemical and physiological basis for effects of controlled and modified atmospheres on fruits and vegetables. Food Technology, Chicago, v.40, n.5, p. 99-104, 1986.

KAYS, S. J. Postharvest physiology of perishable plant products. 
Athens: AVI, 1997. 532p.

KAVATI, R. Pitanga, o sucesso é internacional. Globo Rural, São Paulo, v.11, n.132, p.17, 1996.

JAIME, P.; SALVADOR, M. L.; ORIA, R. Respiration rate of sweet cherries: 'Burlat', 'Sunburst' and 'Sweetheart' cultivars. Journal of Food Science, Chicago, v.66, n.1, p.43-47, 2001.

MORRIS, L. L. Chilling injury of horticultural crops: an overview. HortScience, Alexandria, v.17, n.2, p. 161-162, 1982.

SAEZ, C; OLÍAS, P. R.; OLÍAS, J. M. Quality of strawberries packed with perforated polypropylene. Journal of Food Science, Chicago, v.64, n.4, p.748-751, 1999.

SALUNKHE, D. K.; Wu, M. T. Developments in technology of storage and handling of fresh fruits and vegetables. CRE Critical Reviews in Food Technology, Boca Raton. v.5, n.1, p.15-54, apr. 1974.

SANTOS, A.F.; SILVA, S.M.; MENDONÇA, R.M.N.; SILVA, M.S.; ALVES, R. E.; FILGUEIRAS, H. A. C. Alterações fisiológicas durante a maturação de pitanga (Eugenia uniflora L.). Proceedings of the
Interamerican Society for Tropical Horticulture, Miame, v.46, p.52-57, 2003 .

SANTOS, A. F.; SILVA, S.M.; MENDONÇA, R.M. N.; ALVES, R. E.; FILGUEIRAS, H. A. C. Maturation and ripening changes in Suriname Cherry (Eugenia uniflora L.) fruits. Proceedings of the Interamerican Society for Tropical Horticulture, Miame, v.45, p.4547, 2002.

WALLACE, A. Organic acid synthesis and accumulation in sweet and sour lemon fruit. Proceedings of American Society of Horticultural Science, Beltsville, v.89, p.182-194, 1966.

WILLS, R. B .H.; MCGLASSON, W. B. R.; GRAHAM, D.; LEE, T. H.; HALL, E. G. Postharvest: an introduction to the phisiology and handling of fruits and vegetables. New York: AVI Van Nostrand Reinhold, 1989.325p.

ZAGORY, D; KADER, A. A. Modified atmosfphere packaging of fresh produce. Food Technology, Chicago, v.42, n.9, p.70-74, 1988. 